Available online on 22.06.2020 at http://jddtonline.info
Open Access to Pharmaceutical and Medical Research
unrestricted non-commercial use, provided the original work is properly cited

Open $\odot$ Access

Review Article

\title{
Liposomal drug delivery system as an effective treatment approach for lung cancer
}

\author{
Kinjal Patel*, Devanshi Patel \\ M.B. Patel Science College, Department of Microbiology S.P. University Anand, Gujarat 388001, India
}

\begin{abstract}
Worldwide, cancer is one of the leading causes of mortality and cancer rates are set to increase at alarming rate globally. There are various types of cancer in which the leading type is the lung cancer. In recent years lipid-based carriers, such as liposomes, have successfully encapsulated chemotherapeutic agents ameliorating some toxicity issues, while enhancing the overall therapeutic activity in cancer patients. In addition to this, nanomaterials can help to improved half-life in the body, morphology, for increased drug loading and many other ways. The survey discussed in this review will lead the anticancer therapy and cancer management which will provide the platform to the next generation. Therefore, this critical review includes the therapeutic interventions, liposomes target delivery, active and passive drug loading. Finally, we attempt to summarize the current challenges in nanotherapeutics and provide an outlook on the future of this important field.
\end{abstract}

Keywords: Drug Delivery, Liposomes target Delivery, Nanostructures, Drug loading

Article Info: Received 24 March 2020; Review Completed 29 May 2020; Accepted 04 June 2020; Available online 22 June 2020 Cite this article as:

Patel K, Patel D, Liposomal drug delivery system as an effective treatment approach for lung cancer, Journal of Drug Delivery and Therapeutics. 2020; 10(3-s):367-370 http://dx.doi.org/10.22270/jddt.v10i3-s.4191

*Address for Correspondence:

Kinjal Patel, M.B. Patel Science College, Department of Microbiology S.P. University Anand, Gujarat 388001, India

\section{INTRODUCTION}

According to the World Cancer Report, cancer rates could further increase by $50 \%$ to 15 million new cases in the year 2020. The most prevalent types of cancer are prostate cancer and breast cancer in men and women, respectively. In addition, Lung cancer is the most frequent cause of death for both sexes. As evidenced by the high incidence and mortality rates, novel treatments strategies for this formidable disease are warranted. Cancer is a disease characterized by uncontrollable, irreversible, independent, autonomous, uncoordinated and relatively unlimited and abnormal over growth of tissues Figure 1. Cancer spreads by invasion to the surrounding tissues and by metastasis to distant sites. There are basically two types of cancer one is Benign tumors are not cancer: generally slow growing expansive masses often with a "Pushing margin" and enclosed within a fibrous capsule and another is Malignant tumors are cancer: usually rapidly growing, invading local tissue and spreading to distant sites.

In all the types of cancer one of most leading cancer is lung cancer which is a commonest cancer caused due to tobacco smoke. It was in the observation that the more than 2 million peoples are affected by tobacco smoke in the world. Currently the intravenous administration of chemotherapeutic agents treatment is used as treatment of lung cancer.

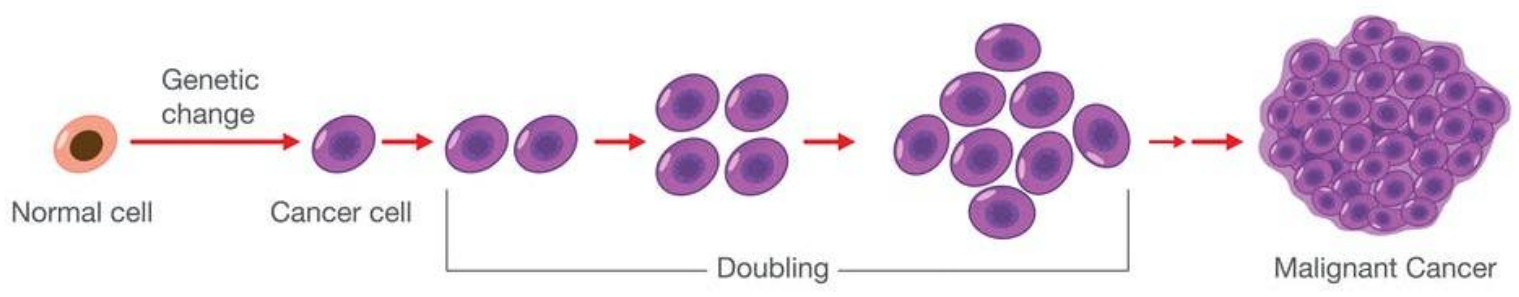

Figure 1 Process of cancer development 


\section{THERAPEUTIC INTERVENTIONS}

Most often cancer is treated with a myriad of therapeutic interventions including surgery, ionizing radiation and chemotherapy, the combination of which depends on the type and stage of disease. The goal of treatment is to reduce local tumor burden and eliminate all malignant cells. Surgery and radiation therapy are effective for local or contained disease, and is often curative at early stages of disease, but not all types of cancer can be treated by these methods.

Treatment of main type of lung cancer which is non-small cell lung cancer/NSCLC generally requires partial surgery along with radiotherapy and chemotherapy. Both, radio and chemotherapy cause painful toxicity to the patient thereby requiring premature end of the therapy leaving the treatment halfway even though the tumor cells are successfully killed. The reason for this is that all cytotoxic drugs kill normal cells as well as cancerous cells resulting in severe side effects. Furthermore, because of the blood circulation in the body, only a small fraction of the drug actually reaches the target tumor tissue and most of the drug acts on normal tissues or is rapidly eliminated. Therefore, to obtain a therapeutic effect, a relatively high dose of drug must be administered and usual drug formulations are used in a balance between killing the tumor (efficacy) and killing the patient by causing lower toxicity to normal organs. The use of combination chemotherapy has been used in current treatment of NSCLC and is associated with a response rate of over $50 \%$ and a median survival of $8-12$ months (1). This chemotherapy comprises only symptomatic management and partial cure. The major problems associated with chemotherapeutic agents are inadequate tumor specificity, narrow therapeutic indices and emergence of resistant cancer cells. Extensive side effects due chemotherapeutic anticancer drugs on normal dividing cells as hair follicles, germ cells and hematopoietic cells are well known resulting in dose limiting toxicity and incomplete therapy.

The continual progress in survival outcomes and advancement in treatments have strongly paralleled the acquired scientific knowledge in tumor biology; and this is highlighted by the development of combination chemotherapy regimens that take into consideration mechanisms of drug action and developmental resistance (2). In addition, recent advances in research using liposomal and nanoparticulate systems in treatment of cancer have led to development of many products for more efficacious means of treating the lung cancer(3). Development of liposomal doxorubicin (DOXIL), daunorubicin, nanoparticulate paclitaxel (Abraxane), monoclonal antibody based Herceptin formulation have been the most successful and effective formulations or treatment of solid tumor and metastatic cancer with higher selectivity at site of cancer and lower systemic side effects. However, direct targeting of these drugs to the lung tissue without systemic side-effects has still been a mystery without any suitable treatment solution.

\section{DRUG DELIVERY BASED ON LIPOSOMES}

Antineoplastic agents used in the treatment of lung cancer have often associated with number of severe toxicities such as bone marrow depression results in granulocytopenia, agranulocytosis, thrombocytopenia, and aplastic anemia, lymphocytopenia and inhibition of lymphocyte function results in suppression of host immunity etc. Currently Paclitaxel, Carboplatin, Cisplatin, Docetaxel, Topotecan, Etoposide, Gemcitabine are the most widely used anticancer agents in treatment of lung cancer with their known reported toxicities. The medications are available as injections for systemic use and result in hazardous side effects due to their non-specificity on the dividing cells in the body. Intracellular transport of different biologically active molecules is one of the key problems in drug delivery in general. Currently the anticancer agents have poor intracellular concentration in the cancer cells.

In view of the light of above facts associated with many of the available chemotherapeutic agents, drug delivery systems have been used as one of the promising strategies to improve pharmacological effects of these drugs. Amongst the many delivery systems designed for intravenous use such as micelles, lipid emulsions, liposomes, polymer-drug conjugates, polymer microspheres, nanoparticles, niosomes, and osmotic pumps, liposome technology has been successful with several products currently available for human use.

\subsection{Liposomes as Drug Carrier}

The use of liposomes as drug delivery agents has evolved from a line of research originating over 40 years ago, based on the ability of these unilamellar vesicles to entrap material in an aqueous compartment (4). It was then known that most amphipathic membrane lipids form multilamellar vesicles (MLV) consisting of concentric bilayers when they are dispersed in aqueous media. MLV are relatively large (micron) sized structures, however they can be extruded through $100 \mathrm{~nm}$ pore size polycarbonate filters to produce unilamellar vesicles with a homogeneous size distribution (5),(6). Typically, the resulting liposomes are $100 \mathrm{~nm}$ in diameter and each particle contains numerous lipid molecules (2). Liposomes have been widely used as models of biological membranes to study membrane permeability and transport across the bilayer (7). In addition to their utility as model membranes, drugs may be encapsulated within their interior aqueous compartment(8). The ability of liposomes to deliver drugs preferentially to disease sites, such as solid tumors, can result in considerable improvements in efficacy; therefore, liposomes are widely studied for use in therapeutic applications Figure 2.
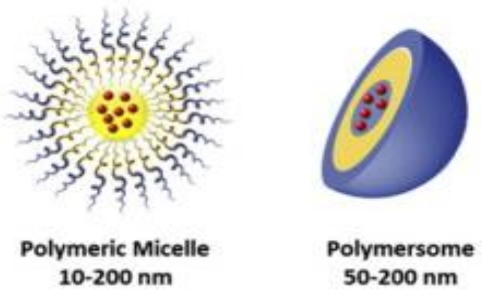

10-200 nm

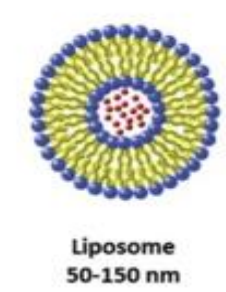

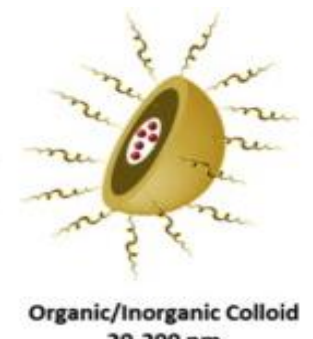

20-200 nm

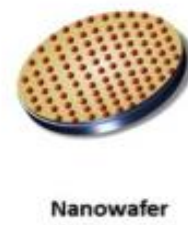

14-500 nm

Figure 2: Types of Liposome for drug Delivery

The first preparation of a liposome with entrapped solute was characterized in 1965 by A. D. Bangham in Cambridge,
UK (4). The evolution of liposomes as drug delivery systems was subsequently accelerated in the $1980, \mathrm{~s}$ by the 
development of techniques to rapidly generate well defined liposomal systems and to efficiently load them with drugs (9),(10). The observation that long-circulating liposomes preferentially accumulate at sites of disease, including sites of infection, inflammation and tumors, due to the leaky nature of the vasculature in these regions(11), (12) gave a solid rationale for delivering drugs in liposomal systems. Liposomes have several features that have contributed to their success as a drug delivery system. Encapsulation within the aqueous cavity of an liposomes can enhance the in vivo activity of drugs by protecting them from breakdown in the body and can reduce the toxic effects of drugs, such as anti-cancer drugs, by reducing delivery to sensitive tissue (13).

\subsection{Liposomes for Targeted Drug Therapy}

The concept of site specific drug delivery for treatment of localized disease in the body to improve therapeutic index of the drug is considered as perennial challenge to the formulator in modern formulation design(14). Constant efforts have been pursued in designing such an ideal drug delivery system which can effectively overcome dose related toxicity and adverse side effects and thus improve patient compliance. One such area which has attracted ever growing attention of pharmaceutical scientist and has shown tremendous potential and promise is colloidal drug carrier system (15). The idea of drug carrier with targeted specificity has fascinated scientists for number of years and in the last decade successful efforts have been made to achieve this goal. The ultimate form of targeted drug delivery system should be realization of Paul Ehrlichs "magic bullet concept" (16) which documents the delivery of drug exclusively to a preselected targeted cell type. Amongst all targeted drug delivery systems, Liposomes are recently gaining popularity because of their biological inert nature, freedom from antigenic, pyrogenic or allergic reaction and their enhanced stability (17).

The development of liposomes containing lipid derivatives of PEG or saturated phospholipids such as DSPC with cholesterol has made targeted liposomal therapy more feasible by reducing the uptake by the RES system and there by prolonging the circulation time.Particularly, PEG is useful because of its ease of preparation, relatively low cost, controllability of molecular weight and linking ability to lipids or peptide including RGD peptide by a variety of methods. The presence of PEG reduces binding of serum protein, i.e. opsonins marking the liposome for clearance by macrophages (18).

Targeting using small peptides like RGD has certain advantages over the use of conventional protein macromolecules. These include ease of preparation, lower antigenicity, and increased stability (19). Antineoplastic agents used in the treatment of lung cancer, solid tumor, testicular cancer, breast cancer, several types of leukemia, lymphoma and etc. have often associated with number of severe toxicities such as bone marrow depression results in granulocytopenia, agranulocytosis, thrombocytopenia, and aplastic anemia, lymphocytopenia and inhibition of lymphocyte function results in suppression of host immunity (20).

\subsection{Drug Loading in Liposomes}

In the early 1970s, it was proposed that liposomes could potentially hold entrapped pharmaceuticals for treatment of diseases. Within this review two anti-cancer drugs, were encapsulated in liposomes. The advantages and disadvantages of different loading methods used to encapsulate these drugs will be discussed below.

\subsubsection{Passive Loading}

Hydrophobic drugs (e.g., taxol and amphotericin B) or water soluble drugs (e.g., cytarabine and gemcitabine) may be passively entrapped within liposomes during hydration of lipid and liposome formation. Encapsulation efficiencies up to $100 \%$ may be achieved for hydrophobic drugs when exhibiting favorable drug-lipid interactions and drug solubility. Passive loading of water soluble drugs is typically very low $(<30 \%)$ and is dependent on the trapped volume of the liposome and liposomal lipid concentration. If drugs have to be encapsulated using passive loading methods it is more difficult to control parameters such as drug-to-lipid ratios and trapping efficiency. In the case of cytotoxic drugs, passive trapping also means that careful methods must be in place during liposome preparation and following preparation to remove the unencapsulated drug.

\subsubsection{Remote Loading}

Drugs, such as anthracycline antibiotics, can alternatively be loaded into preformed liposomes containing a $\mathrm{pH}$ gradient ( $\mathrm{pH} 4.0$ inside, $\mathrm{pH} 7.4$ outside). This method is limited to drugs having an ionizable amine function, and results in encapsulation efficiencies of less than 98\%. For anthracyclines, the encapsulation efficiencies are much higher than predicted by the Henderson-Hasselbach equation, and may be explained by the formation of drug micro precipitates and/or drug association with or partitioning into the lipid membrane. Drug retention by this method is dependent on liposome composition including surface charge, phospholipid acyl chain length, cholesterol content, internal buffering capacity, drugto- lipid ratio, $\mathrm{pH}$ gradient, and liposome size parameters that can all be independently altered. Other active loading methodologies include the ammonium sulfate gradient

methodand metal complexation. The latter is of potential interest since drug loading may not be dependent on maintenance of an established $\mathrm{pH}$ gradient.

\section{CHALLENGES TO DRUG DELIVERY TO SOLID TUMORS}

The term "tumor" (neoplasm) refers to a collection of abnormally growing cells which is not always synonymous with cancer. Some tumors are benign (non-cancerous) while others are malignant (cancerous). The term solid tumor is used to distinguish between a localized solid mass of tissue and leukemia. Leukemia is a type of cancer that is defined by abnormal increase in the number of leukocytes and since it affects the blood, it takes on fluid properties. Malignant tumors differ from benign ones in that they are capable of spreading into surrounding tissues (invasion) and transferring to other organs or parts of the body that are not directly in contact with the malignant tumor through blood circulation and/or lymphatic system (metastasis). Tumors tend to grow exponentially; a single tumor cell can produce a one-gram tumor ( 10 to 10 cells) after about 30 doublings in volume, and with another 10 doublings in volume it would produce a very large tumor of about one kilogram (21),(22). Over $85 \%$ of the human cancers are solid tumors (23).

An elevated interstitial pressure further limits drug diffusion into distant tumor cells (24). The center of the tumor has high interstitial pressure. This elevated pressure in the inner zone can impede movement of drug molecules into the tumor matrix. 6 . In addition, rapidly proliferating cancer cells easily undergo treatment resistant mutations and are known to develop, so called, 'multi drug resistance, very soon. Development of this drug resistance is probably through generation of drug exporters in these tumor cells 
(25). Metastasis of these cancerous cells spreads the disease to other tissues as well. These limitations virtually restrict our ability to kill the solid tumor cancer cells per se with the available cytotoxic drugs; therefore, the actual target has to be redefined. Recently, vasculature targeting has been proposed to be a promising alternative for effective solid tumor treatment (26). This approach involves killing tumor cells by denying them of their life blood. This strategy may involve anti-angiogenesis-inhibition of new blood vessel formation, or anti-vascular approaches that aim to cause a rapid and extensive shutdown of the established tumor vasculature leading to secondary tumor cell death. The functioning vascular network in tumors provides the tumor cells with oxygen and nutrients and enables removal of the toxic waste products of cellular metabolism and as such, it is pivotal for the survival of the tumor cells. Disruption of this vasculature results in tumor cell death and consequently tumor cures. Malignant cells, like normal mammalian cells require oxygen and nutrients for their survival and are, therefore, located in near area.

For tumors to grow beyond this size, they must recruit new blood vessels by vasculogenesis and angiogenesis. Without blood vessels, tumors cannot grow beyond a critical size and cannot metastasize to another organ (27). Considering the limitations of existing solid tumor cancer therapy, antivascular therapy using solid tumor vasculature targeting appears to be an attractive strategy.

\section{CONCLUSION}

Cancer is the deadliest disease and effective treatment to solid tumor such as lung cancer is very challenging. Though there are anti-cancer drugs available for better therapy, there is a need to have a promising drug delivery system which can reduce the side effects and improve the therapy outcome. Liposomes are such drug delivery vesicles in which variety of drugs can be loaded and can be delivered at the tumor site. Researchers are designing liposomal delivery systems to deliver various classes of drugs for the cancer treatment and that has a commercial application too. To conclude, a lot of research have been done in studying liposomes as a drug carrier for cancer therapy and may serve as an upcoming strategy for efficient treatment of lung cancer.

\section{REFERENCES}

1. Aisner J, Goutsou M, Maurer LH, Cooper R, Chahinian P, Carey R, et al. Intensive combination chemotherapy, concurrent chest irradiation, and warfarin for the treatment of limited-disease small-cell lung cancer: a Cancer and Leukemia Group B pilot study. Journal of clinical oncology : official journal of the American Society of Clinical Oncology. 1992;10(8):1230-6.

2. Winterhalter M, Lasic DD. Liposome stability and formation: experimental parameters and theories on the size distribution. Chemistry and physics of lipids. 1993;64(1-3):35-43.

3. Patel J, Amrutiya J, Bhatt P, Javia A, Jain M, Misra A. Targeted delivery of monoclonal antibody conjugated docetaxel loaded PLGA nanoparticles into EGFR overexpressed lung tumour cells. Journal of microencapsulation. 2018;35(2):204-17.

4. Bangham AD, Standish MM, Watkins JC. Diffusion of univalent ions across the lamellae of swollen phospholipids. Journal of molecular biology. 1965;13(1):238-52.

5. Olson F, Hunt CA, Szoka FC, Vail WJ, Papahadjopoulos D. Preparation of liposomes of defined size distribution by extrusion through polycarbonate membranes. Biochimica et biophysica acta. 1979;557(1):9-23.

6. Szoka F, Jacobson K, Derzko Z, Papahadjopoulos D. Fluorescence studies on the mechanism of liposome-cell interactions in vitro. Biochimica et biophysica acta. 1980;600(1):1-18.
7. Madden TD. Chapter 1 Model membrane systems. In: Bittar EE, Bittar N, editors. Principles of Medical Biology. 7: Elsevier; 1997. p. 1-17.

8. Bhatt P, Lalani R, Vhora I, Patil S, Amrutiya J, Misra A, et al. Liposomes encapsulating native and cyclodextrin enclosed paclitaxel: Enhanced loading efficiency and its pharmacokinetic evaluation. International journal of pharmaceutics. 2018; 536(1):95-107.

9. Mayer LD, Bally MB, Hope MJ, Cullis PR. Uptake of antineoplastic agents into large unilamellar vesicles in response to a membrane potential. Biochimica et biophysica acta. 1985;816(2):294-302.

10. Gabizon A, Goren D, Fuks Z, Meshorer A, Barenholz Y. Superior therapeutic activity of liposome-associated adriamycin in a murine metastatic tumour model. Br J Cancer. 1985;51(5):6819.

11. Dean RT. Lysosomes and membrane recycling. A hypothesis. Biochemical Journal. 1977;168(3):603-5.

12. Jain MK, Zakim D. The spontaneous incorporation of proteins into preformed bilayers. Biochimica et biophysica acta. 1987;906(1):33-68.

13. Forssen EA, Tökès ZA. Use of anionic liposomes for the reduction of chronic doxorubicin-induced cardiotoxicity. Proceedings of the National Academy of Sciences of the United States of America. 1981;78(3):1873-7.

14. Bhatt P, Vhora I, Patil S, Amrutiya J, Bhattacharya C, Misra A, et al. Role of antibodies in diagnosis and treatment of ovarian cancer: Basic approach and clinical status. Journal of controlled release : official journal of the Controlled Release Society. 2016;226:148-67.

15. Akbarzadeh A, Rezaei-Sadabady R, Davaran S, Joo SW, Zarghami $\mathrm{N}$, Hanifehpour $\mathrm{Y}$, et al. Liposome: classification, preparation, and applications. Nanoscale Res Lett. 2013;8(1):102-.

16. Vyas SP, Sihorkar V, Mishra V. Controlled and targeted drug delivery strategies towards intraperiodontal pocket diseases. Journal of clinical pharmacy and therapeutics. 2000;25(1):2142.

17. Mishra PK, Gulbake A, Jain A, Vyas SP, Jain SK. Targeted delivery of an anti-cancer agent via steroid coupled liposomes. Drug delivery. 2009;16(8):437-47.

18. Patel P, Hanini A, Shah A, Patel D, Patel S, Bhatt P, Pathak YV. Surface Modification of Nanoparticles for Targeted Drug Delivery. In Surface Modification of Nanoparticles for Targeted Drug Delivery ,Cham: Springer International Publishing. 2019:19-31.

19. Lalani RA, Bhatt P, Rathi M, Misra A. Abstract 2063: Improved sensitivity and in vitro efficacy of RGD grafted PEGylated gemcitabine liposomes in RRM1 siRNA pretreated cancer cells. Cancer Research. 2016;76(14 Supplement):2063.

20. Tripathi D, Therapondos G, Lui HF, Johnston N, Webb DJ, Hayes PC. Chronic Administration of Losartan, an Angiotensin II Receptor Antagonist, is not Effective in Reducing Portal Pressure in Patients with Preascitic Cirrhosis. American Journal of Gastroenterology. 2004;99(2).

21. Tannock IF, Warr DG. Unconventional therapies for cancer: a refuge from the rules of evidence? CMAJ : Canadian Medical Association journal de l'Association medicale canadienne. 1998;159(7):801-2.

22. Tannock IF. Conventional cancer therapy: promise broken or promise delayed? Lancet (London, England). 1998;351 Suppl 2:Sii9-16.

23. Jang SH, Wientjes MG, Lu D, Au JL. Drug delivery and transport to solid tumors. Pharmaceutical research. 2003;20(9):1337-50.

24. Baxter LT, Jain RK. Transport of fluid and macromolecules in tumors. I. Role of interstitial pressure and convection. Microvascular Research. 1989;37(1):77-104.

25. Doyle L, Ross DD. Multidrug resistance mediated by the breast cancer resistance protein BCRP (ABCG2). Oncogene. 2003;22(47):7340-58.

26. Molema G. Tumor vasculature directed drug targeting: applying new technologies and knowledge to the development of clinically relevant therapies. Pharmaceutical research. 2002;19(9):1251-8.

27. Carmeliet P. Angiogenesis in health and disease. Nature medicine. 2003;9(6):653-60 